\title{
DIAGNÓSTICO DE PROBLEMAS RELACIONADOS CON MEDICAMENTOS EN ADULTOS MAYORES AL MOMENTO DE SER HOSPITALIZADOS
}

\author{
Teodoro J. Oscanoa1,a
}

\begin{abstract}
RESUMEN
Objetivos. Diagnosticar los Problemas Relacionados con Medicamentos (PRM) en pacientes mayores de 64 años al momento de ser hospitalizados. Materiales y métodos. La detección de sobreprescripción y disprescripción de medicamentos se realizó con el Índice de Uso Apropiado de Medicamentos. Para subprescripcion se usaron los indicadores de Uso Adecuado de Medicamentos en adultos mayores vulnerables y la prueba de Evaluación de Subutilizacion de Medicamentos. La valoración de la adherencia a la medicación y de las reacciones adversas medicamentosas se realizó con el cuestionario de Morisky-Green y el Algoritmo de Karch y Lasagna, respectivamente. Resultados. El estudio se realizó en 100 pacientes del servicio de geriatría de un hospital de alta complejidad en Lima Perú; la media de edad fue de 76,26 \pm 6,$91 ; 55 \%$ de sexo masculino. Al evaluar 555 fármacos con el Índice de Uso Apropiado de Medicamentos, 254 (45,8\%) tuvieron al menos uno o más de los criterios de prescripción inadecuada, lo que correspondió a 89 pacientes estudiados. Se encontró subutilización de medicamentos en pacientes que debieron recibir betabloqueadores por antecedente de infarto agudo de miocardio (59\%) y warfarina o aspirina por la condición de fibrilación auricular (21\%). La no adherencia y la frecuencia de reacciones adversas que motivaron la hospitalización fueron de $63 \%$ y $24 \%$, respectivamente. Conclusiones. El diagnóstico de los PRM en adultos mayores al momento de ser hospitalizados utilizando instrumentos validados, es de gran utilidad y debería formar parte de la valoración geriátrica integral en el adulto mayor.
\end{abstract}

Palabras clave: Prescripciones de medicamentos; Evaluación de medicamentos; Adultos mayores débiles (fuente: DeCS BIREME).

\section{DIAGNOSIS OF DRUG-RELATED PROBLEMS IN ELDERLY PATIENTS AT THE TIME OF HOSPITALIZATION}

\begin{abstract}
Objectives. To diagnose drug-related problems (DRPs) in patients over 64 years of age at the time of hospitalization. Materials and methods. The detection of overprescription and misprescription of medications was done using the Index of Appropriate Drug Use, and for underprescription the Indication of Adequate Drug Use in Vulnerable Elderly Adults and the test of the Evaluation of the Underuse of Drugs were used. The evaluation of drug adherence and adverse drug reactions was completed using the Morisky-Green Questionnaire and the Karch and Lasgna Algorithm, respectively. Results. The study was conducted in 100 inpatients in a geriatric service in a multi-specialty hospital in Lima, Peru; the median age was $76.26 \pm 6.91$ and $55 \%$ were males. After evaluating 555 drugs with the Index of Appropriate Drug Use, 254 of them $(45.8 \%)$ qualified for at least one or more of the criteria studied for inadequate prescription, corresponding to 89 of the studied patients. We found the underuse $(21 \%)$ of Warfarin or aspirin by eligible patients with auricular fibrillation, and no use (59\%) of beta blockers by patients with a history of acute myocardial infarction. The non-adherence and frequency of adverse reactions that motivated hospitalization were $63 \%$ and $24 \%$, respectively. Conclusions. The diagnosis of DRPs in elderly adults at the time of hospitalization using valid instruments is extremely useful and should be part of the integral geriatric evaluation of the elderly.
\end{abstract}

Key words: Drug prescriptions; Drug evaluation; Frail elderly (source: MeSH NLM).

\section{INTRODUCCIÓN}

La regla de oro de una intervención farmacológica siempre fue "fármaco indicado, paciente correcto y tiempo adecuado" (1).Sin embargo, en los pacientes geriátricos donde la farmacocinética y farmacodinámica de los medicamentos es modificada de manera significativa por el proceso de envejecimiento y la susceptibilidad a los eventos adversos de los medicamentos, se incrementa; a la regla de oro mencionada se debe agregar: "ausencia de Problemas Relacionados con Medicamentos (PRM)". Se define PRM a problemas de salud, entendidos como resultados clínicos negativos, derivados de la farmacoterapia que, producidos por diversas causas, conducen a la no consecución del objetivo terapéutico o a la aparición de efectos no deseados ${ }^{(2)}$. Para fines

Servicio de Geriatría del Hospital Nacional Guillermo Almenara Irigoyen. Lima, Perú.

a Médico Internista, Doctor en Medicina.

Recibido: 14-02-11 Aprobado: 01-06-11 
prácticos pueden ser comparados como enfermedades o problemas, que bajo la óptica médica, deben ser diagnosticados, intervenidos e idealmente prevenidos. Los pacientes geriátricos, debido a sus múltiples comorbilidades, son polimedicados, por lo que es una tarea imprescindible la revisión sistemática y periódica de sus fármacos habituales.

La evaluación de la calidad de prescripción farmacológica y la detección de PRM en un paciente particular tiene que evidenciar cinco fenómenos de prescripción farmacológica: sobreprescripción, disprescripción, subuso de fármacos, detección de reacciones adversas medicamentosas y valoración de la adherencia o cumplimiento terapéutico. Se denomina sobreprescripción de medicamentos a la prescripción de más medicamentos que los clínicamente necesarios ${ }^{(3,4)}$. Disprescripción es la prescripción incorrecta de un fármaco que tiene una indicación clínica, los errores pueden ser selección del fármaco, dosis, forma de administración, duración de la terapia, interacción medicamentosa, monitorización y costo ${ }^{(3,4)}$.La subutilización de medicamentos es la omisión de una intervención farmacológica de probada efectividad según criterios de Medicina Basada en Evidencias, existiendo la condición o enfermedad que exigía tal intervención terapéutica ${ }^{(5)}$.

La presente investigación tiene por objetivo diagnosticar los Problemas Relacionados con Medicamentos (PRM) en pacientes mayores de 64 años al momento de ser hospitalizados, utilizando pruebas validadas previamente en la literatura médica.

\section{MATERIALES Y MÉTODOS}

El presente estudio es del tipo observacional, descriptivo, de serie de casos. La investigación se llevó a cabo en el Servicio de Geriatría del Hospital Almenara en Lima Perú entre el 1 de enero y el 30 de abril de 2009. Los criterios de inclusión para el estudio fueron pacientes mayores de 64 años y medicación habitual mayor de 1 . Se denominó medicación habitual, a aquel fármaco que el paciente refería estar tomando (también se incluyó la referencia del cuidador o familiar) por más de 15 días antes de ser hospitalizado. Durante el periodo del estudio se hospitalizaron en la Unidad Geriátrica de Agudos del Servicio de Geriatría, 212 pacientes, de los cuales ingresaron al estudio 100 pacientes. Se excluyó a 112 por tener menos de 65 años (el criterio de hospitalización en el servicio es de pacientes mayores de 60 años), estar tomando menos de dos fármacos y aquellos en los cuales no se logró la identificación clara de fármacos referidos como medicación habitual.

\section{VALORACIÓN GERIÁTRICA INTEGRAL}

Durante las primeras 24 horas de hospitalización un médico residente de Geriatría elaboró la historia clínica geriátrica completa. En ella, se consignaron datos demográficos, examen físico, valoración geriátrica integral que incluye la valoración de las actividades de la vida diaria mediante el índice de Katz ${ }^{\left({ }^{6}\right)}$, evaluación estado cognitivo mediante el test de Folstein o Minimental Test ${ }^{(7)}$ y el índice de comorbilidad de Charlson ${ }^{(8)}$.

\section{INFORMACIÓN SOBRE MEDICACIÓN HABITUAL}

El estudio fue enfocado a la detección de los PRM de medicamentos habituales tomados, previos a la hospitalización, por un tiempo no menor de 15 días, ello a través de una entrevista con el paciente o con el cuidador/familiar. También se revisó recetas o formularios expedidos por prescriptores; en caso de automedicación, se anotó la forma y posología con que el paciente solía tomar los medicamentos. Adicionalmente, se revisó la historia clínica antigua de aquellos pacientes con antecedentes de haber sido atendidos en el Hospital Almenara. Se tomó en cuenta las notas de transferencias de centros periféricos y hospitales, especialmente los relacionados con prescripción de medicamentos. Se utilizó la estrategia de la "bolsa de compras" para identificar todos los medicamentos habituales; esta consiste en recomendar al paciente o cuidador traer en una bolsa todos los medicamentos que consumía hasta el momento de ser hospitalizado, posteriormente se pregunta por su uso e indicación.

\section{METODOLOGÍA DIAGNÓSTICA DE PRM}

Para la detección de PRM catalogados como sobreprescripción y disprescripción se utilizó el Índice de Uso Apropiado de Medicamentos (Medication Appropriateness Index o MAI) ${ }^{(9)}$. En relación con las características clinimétricas del MAI ${ }^{(9)}$, su coeficiente de variabilidad interobservador para señalar lo apropiado de un medicamento en un paciente es de 0,88 y para señalar lo inadecuado de una prescripción medicamentosa es de 0,95 para un valor kappa total de 0,83 . Por otro lado, el coeficiente de variabilidad intraobservador, para la calificación de lo adecuado de una prescripción medicamentosa fue de 0,94 y para señalar lo inapropiado de una medicación específica fue de 0,98 para un valor kappa total de 0,92 .

Se utilizó el MAI de acuerdo con el procedimiento descrito por Hanlon y col. ${ }^{(9)}$. El desarrollo del MAI consiste en la calificación de las siguientes características principales de una prescripción (entre paréntesis se consigna los ponderados asignados a cada ítem cuando este 
es considerado inadecuado): Indicación (3), efectividad (3), dosis (2), instrucciones correctas (2), instrucciones prácticas (1), interacción farmacológica (2), interacción fármaco-enfermedad (2), redundancia innecesaria (1), duración (1) y costo (1). Para la aplicación del instrumento se tomó en cuenta que la institución donde se realizó (ESSALUD) el estudio, solo atiende pacientes asegurados y tiene un petitorio farmacológico (10); por consiguiente, cualquier fármaco prescrito y no incluido dentro de la lista de fármacos, fue comprado por el paciente y se investigó la existencia de una alternativa farmacológica igualmente efectiva y segura, pero incluida en dicho petitorio. Al final de la evaluación de cada fármaco se suman las calificaciones en cada uno de los diez criterios, pudiéndose obtener puntajes que van del 0 a 18. Una prescripción adecuada en todos los criterios señalados totaliza 0 . Las sumatorias mayores de 0 , son interpretadas como prescripción inadecuada; este será mayor cuanto más criterios analizados sean calificados como inadecuados. Puede considerarse que el desarrollo del MAI, es fundamentalmente una auditoría de la prescripción farmacológica, teniendo como referencia las Guías de Práctica Clínica actualizadas sobre la farmacoterapia específica de cada caso evaluado. El desarrollo de MAI en cada uno de los fármacos de los pacientes estudiados estuvo a cargo de un médico especialista en medicina interna y geriatría. Se empleó el VA Medication Classification System (11) para clasificar cada uno de los fármacos consumidos por los pacientes.

Para la detección de no adherencia se utilizó el Cuestionario de Morisky y Green ${ }^{(12)}$ validada al español por Val Jiménez y col. ${ }^{(13)}$. Dicho cuestionario fue aplicado directamente al paciente $o$ al cuidador/familiar, dependiendo de la presencia de trastorno del sensorio o trastorno cognitivo. Se consideró paciente no adherente cuando el puntaje obtenido fue mayor de cero.

Para la evaluación de subuso de fármacos se utilizó 17 indicadores de Uso Adecuado de Medicamentos en Adultos Mayores Vulnerables (Assessing Care of Vulnerable Elder o ACOVE) ${ }^{(14)}$. La calificación del cumplimiento o no, del indicador (subutilización) se hizo exclusivamente en los pacientes considerados elegibles para la terapia farmacológica. Se consideró no elegible cuando no estaba presente la condición o enfermedad y también cuando existía pero había una contraindicación para la administración del fármaco. En las condiciones o enfermedades no consignados en ACOVE, se utilizó la metodología descrita por Lipton y col. denominada Evaluación de Subutilización de Medicamentos (Assessment of Underutilization of Medication) (15). Para el establecimiento de una relación causa-efecto de una reacción adversa medicamentosa se empleó el algoritmo

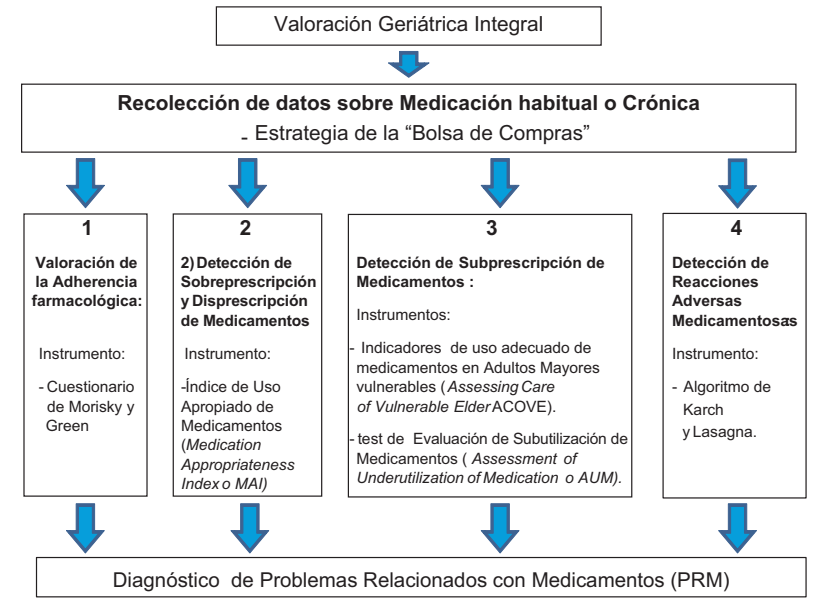

Figura 1. Metodología diagnóstica de Problemas Relacionados con Medicamentos (PRM) en adultos mayores al momento de ser hospitalizados.

de Karch y Lasagna ${ }^{(16)}$. La Figura 1 muestra gráficamente la Metodología Diagnóstica de Problemas Relacionados con Medicamentos (PRM) usada en el estudio.

\section{ANÁLISIS ESTADÍSTICO}

Para el análisis estadístico se tomó en cuenta las variables demográficas (edad, sexo, grado de instrucción), variables relacionadas a evaluación geriátrica integral (número de actividades de la vida diaria dependientes, índice de Charlson y test de Folstein o Minimental Test). Se utilizó el programa bioestadístico EPI INFO 2008 versión 3.5.1.

\section{ASPECTOS ÉTICOS}

La detección de un problema relacionado con medicamentos fue seguido de la comunicación al médico tratante y, en los casos necesarios, se procedió a las interconsultas a las especialidades pertinentes. En los casos en que se detectó reacciones adversas y se decidió la suspensión de la medicación, se tuvo en cuenta los protocolos de suspensión de fármacos para evitar una reacción adversa por retiro inadecuado de fármacos (17). La valoración y detección de casos de subutilización siguió el mismo procedimiento descrito previamente con los PRM.

\section{RESULTADOS}

Las características demográficas de los 100 pacientes se describen en la Tabla 1. Los pacientes incluidos presentaron un índice de comorbilidad de Charlson de $3,5 \pm 2,2$ (rango: 0-11) y la media del número 
Tabla 1. Características clínicas y demográficas de la población estudiada

\begin{tabular}{|c|c|}
\hline Características demográficas & Valores \\
\hline Edad (media $\pm D E)$ & $76,3 \pm 6,9$ \\
\hline $\begin{array}{l}\text { Grupos etarios: } \\
65-74 \text { años } \\
75-85 \text { años } \\
>85 \text { años }\end{array}$ & $\begin{array}{l}39(39 \%) \\
50(50 \%) \\
11(11 \%)\end{array}$ \\
\hline $\begin{array}{l}\text { Sexo: } \mathrm{n}(\%) \\
\text { Masculino } \\
\text { Femenino }\end{array}$ & $\begin{array}{l}55(55 \%) \\
45(45 \%)\end{array}$ \\
\hline \multicolumn{2}{|l|}{ Escolaridad n (\%) } \\
\hline $\begin{array}{l}\text { Analfabetos } \\
\text { Primaria } \\
\text { Secundaria } \\
\text { Superior }\end{array}$ & $\begin{array}{c}5(5 \%) \\
48(48 \%) \\
29(29 \%) \\
18(18 \%)\end{array}$ \\
\hline Características clínicas & Valores \\
\hline Número total de fármacos del grupo investigado & 555 \\
\hline $\begin{array}{l}\text { Número de fármacos por paciente } \\
\text { (media y desviación típica) }\end{array}$ & $5,6 \pm 1,5$ \\
\hline Mediana de número de fármacos por paciente & 5 \\
\hline \multicolumn{2}{|l|}{ Número de Fármacos: distribución } \\
\hline $\begin{array}{l}2 \text {-4 fármacos } \\
4 \text {-5 fármacos } \\
>5 \text { fármacos }\end{array}$ & $\begin{array}{l}15(15 \%) \\
75(75 \%) \\
10(10 \%)\end{array}$ \\
\hline Índice de Charlson & $3,5 \pm 2,2$ \\
\hline $\begin{array}{l}\text { Número de deficiencias en las actividades de la } \\
\text { vida diaria (alimentación, continencia, transfe- } \\
\text { rencia, uso del retrete, vestir, baño) (media } \pm E \text { ) }\end{array}$ & $3,6 \pm 2,2$ \\
\hline \multicolumn{2}{|l|}{ Actividades de la vida diaria: Índice de Katz } \\
\hline $\begin{array}{l}A \\
B \\
C \\
D \\
E \\
F \\
G\end{array}$ & $\begin{array}{c}13(13 \%) \\
11(11 \%) \\
11(11 \%) \\
16(16 \%) \\
6(6 \%) \\
10(10 \%) \\
33(33 \%)\end{array}$ \\
\hline $\begin{array}{l}\text { Valoración Miniexamen } \\
\text { cognoscitivo de Lobo (media } \pm D E)\end{array}$ & $21,6 \pm 8,4$ \\
\hline $\begin{array}{l}\text { Puntaje } 24 \text { a } 30 \\
\text { Menor de } 24 \\
\text { No se evaluó cognitivamente por trastorno } \\
\text { del sensorio }\end{array}$ & $\begin{array}{c}43(43 \%) \\
41(41 \%) \\
5(4 \%)\end{array}$ \\
\hline
\end{tabular}

de deficiencias en las actividades de la vida diaria (alimentación, continencia, transferencia, uso del retrete, vestir, baño) fue 3,6 $\pm 2,2$ (rango: 0-6). Solo 13 pacientes presentaron índice de Katz A, 87 fueron dependientes en al menos una actividad de la vida diaria y 33 lo fueron en todas. La evaluación cognitiva con el Minimental Test encontró una media de puntaje $21,6 \pm 8,4$ (rango: 0-30); 43 pacientes tuvieron puntaje de 24 a 30; 41 pacientes obtuvieron menos de 24; en cinco pacientes no se evaluó la cognición por trastorno del sensorio. Durante el periodo de hospitalización diez pacientes fallecieron.

La media del número de fármacos por paciente fue de 5,6 $\pm 1,5$ (rango: $2-9$ ); 75 pacientes consumían entre cuatro a cinco fármacos y diez de ellos más de cinco. La media de fármacos al momento de alta fue de 4,38 $\pm 1,45$ (rango: 2-8). La totalidad de fármacos analizados utilizando los criterios del instrumento Índice de Uso Apropiado de Medicamentos fue de 555 . En 89 pacientes se evidenció al menos un criterio considerado inadecuado en su medicación habitual; por otro lado, del total de medicamentos evaluados, $254(45,8 \%)$ tuvieron al menos uno o más de los criterios estudiados calificados como de prescripción inadecuada.

Las cinco clases de fármacos, según la clasificación VA Medication Classification System (11), con mayor frecuencia de prescripción inadecuada fueron los medicamentos cardiovasculares $(35,4 \%)$, sistema nervioso central $(15,4 \%)$, gástrico $(15,4 \%)$, musculoesqueléticos $(8,3 \%)$ y productos hematológicos $(7,1 \%)$ (Tabla 2$)$. Los PRM, detectados con el Índice de Uso Apropiado de Medicamentos catalogados como de sobreprescripción y disprescripción más frecuentes fueron: duración de farmacoterapia no aceptable o no determinada (112 fármacos), costo más elevado que alternativa (65 fármacos), dosis incorrecta (53 fármacos) e instrucciones incorrectas (50 fármacos) (Tabla 3). Los medicamentos más frecuentes con puntajes considerados inadecuados en los diferentes ítems del instrumento fueron: nimodipino, ranitidina, aspirina dosis mayor de $500 \mathrm{mg} / \mathrm{día}$, digoxina y warfarina. Debe mencionarse que solo el $2,2 \%$ de los medicamentos estudiados no estaban incluidos en el petitorio farmacológico de ESSALUD, entre los cuales figuraban en orden decreciente: multivitamínicos, fenazopiridina, pentoxifilina, hidergina y piracetam.

Los porcentajes de pacientes elegibles para una condición o enfermedad donde ocurrió un PRM del tipo subprescripción de medicamentos fueron con mayor frecuencia con: warfarina o aspirina en pacientes con fibrilación auricular $(21 \%)$, beta bloqueadores para pacientes con antecedentes de infarto agudo de miocardio $(58,82 \%)$, inhibidores de la enzima angiotensina convertasa en diabético con proteinuria (33\%), ácido acetilsalicílico diaria para diabético $(27 \%)$ e inhibidores de la enzima angiotensina convertasa para pacientes con insuficiencia cardiaca (10\%) (Tabla 4).

El porcentaje de pacientes adherentes al tratamiento farmacológico fue del $37 \%$. La no adherencia se correlacionó con el mayor número de fármacos $(p<0,001)$, no siendo significativa su correlación con variables como sexo, edad, trastorno cognitivo (Folstein<24), índice de 
Tabla 2. Clases de fármacos con problemas de sobreprescripción y disprescripción según criterios del Medication Appropriateness Index (MAl)

\begin{tabular}{lcc}
\hline $\begin{array}{l}\text { Clases de Fármacos } \\
\text { (VA Medication Classification System) }\end{array}$ & $\begin{array}{c}\text { Frecuencia de } \\
\text { fármacos n (\%) }^{*}\end{array}$ & $\begin{array}{c}\text { Pacientes }^{\dagger} \\
\mathbf{n}(\%)\end{array}$ \\
\hline $\begin{array}{l}\text { Cardiovascular (antihipertensivos, inotrópicos, antianginosos, } \\
\text { hipolipemiantes) }\end{array}$ & $90(35,40 \%)$ & $60(60 \%)$ \\
Sistema nervioso central (anticonvulsivos, antiparkinsonianos, & $39(15,40 \%)$ & $27(27 \%)$ \\
antipsicóticos, ansiolíticos, hipnóticos) & $39(15,40 \%)$ & $28(28 \%)$ \\
Gástrico (drogas antiulcerosas, antiácidos) & $21(8,30 \%)$ & $18(18 \%)$ \\
Musculoesqueléticos (analgésicos y antiinflamatorios no esteroideos) & $18(7,10 \%)$ & $16(16 \%)$ \\
Productos hematológicos/modificadores/expansores volumen & $15(5,90 \%)$ & $13(13 \%)$ \\
(anticoagulantes) & $6(2,40 \%)$ & $6(6 \%)$ \\
Hormonas/sintéticos/Modificadores & $6(2,40 \%)$ & $5(5 \%)$ \\
Antimicrobianos (antibacterianos) & $4(1,60 \%)$ & $4(4 \%)$ \\
Vitaminas & $4(1,60 \%)$ & $3(3 \%)$ \\
Genitourinario & $4(1,60 \%)$ & $3(3 \%)$ \\
Agentes respiratorios & $3(1,20 \%)$ & $3(3 \%)$ \\
Nutrientes terapéuticos/minerales/electrolitos & $3(1,20 \%)$ & $2(2 \%)$ \\
Antihistamínicos & $1(0,40 \%)$ & $1(1 \%)$ \\
Agentes oftalmológicos & $1(0,40 \%)$ & $1(1 \%)$ \\
Agentes dermatológicos & 254 & $100(100 \%)$ \\
\hline Miscelánea & &
\end{tabular}

* Total de fármacos con problemas de sobreprescripción y disprescripción. Los porcentajes están en relación con el total (254).

$\dagger$ Un paciente puede haber recibido más de una clase de medicamentos.

Tabla 3. Problemas relacionados con medicamentos de tipo sobreprescripción y disprescripcion de medicamentos en adultos mayores, Instrumento utilizado: Índice de Uso Apropiado de Medicamentos (MedicationAppropriatenessIndex, MAl) (9)

\begin{tabular}{|c|c|c|}
\hline $\begin{array}{l}\text { Problema relacionado con } \\
\text { medicamentos }\end{array}$ & $\begin{array}{c}\text { Fármacos } \\
\mathrm{n}(\%)^{*}\end{array}$ & Fármacos más frecuentes \\
\hline $\begin{array}{l}\text { Criterio 1: no existencia de indicación } \\
\text { activa }\end{array}$ & $39(7,03 \%)$ & $\begin{array}{l}\text { Ranitidina, nimodipino, aspirina dosis mayor de } 500 \mathrm{mg} / \mathrm{día} \text { y } \\
\text { diazepam. }\end{array}$ \\
\hline $\begin{array}{l}\text { Criterio2: fármaco inefectivo para la } \\
\text { condición }\end{array}$ & $46(8,29 \%)$ & Nimodipino, nifedipino acción corta y diazepam \\
\hline Criterio 3: dosis incorrecta & $53(9,55 \%)$ & Digoxina, warfarina y glibenclamida. \\
\hline Criterio 4: instrucciones no correctas & $50(9,01 \%)$ & Warfarina, digoxina, captopril, enalapril, glibenclamida y haloperidol \\
\hline $\begin{array}{l}\text { Criterio } 5 \text { : Indicaciones no prácticas } \\
\text { en la receta }\end{array}$ & $17(3,06 \%)$ & Captopril, digoxina, enalapril, glibenclamida y haloperidol. \\
\hline $\begin{array}{l}\text { Criterio 6: interacción medicamentosa } \\
\text { clínicamente significativa }\end{array}$ & $21(3,78 \%)$ & $\begin{array}{l}\text { Verapamilo - digoxina (bradicardia) } \\
\text { Espironolacotona - enalapril (hiperpotasemia) } \\
\text { Warfarina - AINEs: Hemorragia digestiva alta. }\end{array}$ \\
\hline $\begin{array}{l}\text { Criterio } 7 \text { :interacción fármaco enfer- } \\
\text { medad }\end{array}$ & $18(3,24 \%)$ & $\begin{array}{l}\text { Enfermedad ulceropéptica - antiinflamatorios no esteroideos. } \\
\text { Calcioantagonistas - estreñimiento, } \\
\text { Metformina - Insuficiencia renal crónica moderada a severa }\end{array}$ \\
\hline $\begin{array}{l}\text { Criterio 8: redundancia innecesaria } \\
\text { con otro medicamento }\end{array}$ & $18(3,24 \%)$ & $\begin{array}{l}\text { Ibuprofeno + ketorolaco, } \\
\text { Naproxeno + ketorolaco } \\
\text { Salbutamol jarabe + salbutamol inhalador }\end{array}$ \\
\hline $\begin{array}{l}\text { Criterio 9: duración de terapia no } \\
\text { aceptable o no determinada }\end{array}$ & $112(20,18 \%)$ & $\begin{array}{l}\text { Ranitidina, haloperidol, diazepam, hidróxido de aluminio, clorfenira- } \\
\text { mina, fenazopiridina, ketorolaco. }\end{array}$ \\
\hline $\begin{array}{l}\text { Criterio 10: costo más elevado que } \\
\text { alternativa }\end{array}$ & $65(11,71 \%)$ & Nimodipino, bromhexina, hidergina, vitamina $\mathrm{C}$ y pentoxifilina \\
\hline
\end{tabular}

* Total medicamentos evaluados: 555 
Tabla 4. Frecuencia de problemas relacionados con subprescripción de medicamentos en adultos mayores. Instrumento utilizado: indicadores de uso adecuado de medicamentos en adultos mayores vulnerables (AssessingCare of Vulnerable Elder) ${ }^{(14)}$

\begin{tabular}{|c|c|c|}
\hline Descripción del Indicador & $\begin{array}{l}\text { Pacientes } \\
\text { elegibles }\end{array}$ & $\begin{array}{l}\text { No Cumplimiento del indicador } \\
\text { (subprescripción) } n(\%)\end{array}$ \\
\hline $\begin{array}{l}\text { Inhibidores de la enzima angiotensina convertasa en diabético con pro- } \\
\text { teinuria }\end{array}$ & 24 & $8(33,33)$ \\
\hline Calcio y vitamina D para pacientes con osteoporosis. & 11 & $3(27,27)$ \\
\hline AAS (ácido acetilsalićlico) diaria para diabético & 22 & $6(27,27)$ \\
\hline $\begin{array}{l}\text { Terapia con fármacos hipolipemiantes para pacientes con enfermedad } \\
\text { cardiaca isquémica con colesterol LDL }>3,4 \mathrm{mmol} / \mathrm{L}(>130 \mathrm{mg} / \mathrm{dL}) \text { sin res- } \\
\text { puesta a dieta. }\end{array}$ & 16 & $8(50,00)$ \\
\hline Betabloqueador para pacientes con falla cardiaca & 10 & $4(40,00)$ \\
\hline Betabloqueador para paciente que haya tenido infarto agudo de miocardio & 17 & $10(58,82)$ \\
\hline $\begin{array}{l}\text { Inhibidores de la enzima angiotensina convertasa para paciente con hi- } \\
\text { pertensión e insuficiencia renal. }\end{array}$ & 16 & $4(25,00)$ \\
\hline Medicación para HTA si no hubo respuesta a la terapia no farmacológica & 61 & $5(8,20)$ \\
\hline $\begin{array}{l}\text { Inhibidores de la enzima angiotensina convertasa para pacientes con in- } \\
\text { suficiencia cardiaca. }\end{array}$ & 20 & $2(10,00)$ \\
\hline Aspirina para paciente con enfermedad cardiaca coronaria & 17 & $3(17,65)$ \\
\hline Prevención de estreñimiento para pacientes que toman opiáceos & 1 & $1(100,00)$ \\
\hline $\begin{array}{l}\text { Warfarina o aspirina, si fuera apropiado, para pacientes con fibrilación } \\
\text { auricular. }\end{array}$ & 33 & $7(21,21)$ \\
\hline
\end{tabular}

Katz, índice de Charlson (Tabla 5). Las tres reacciones adversas medicamentosas más frecuentes que produjeron hospitalización fueron: sangrado asociado a sobreanticoagulación con warfarina, hemorragia digestiva alta inducida por antiinflamatorios no esteroideos e hipoglicemia inducida por fármacos (Tabla 6).

El tiempo promedio para aplicar el instrumento Uso

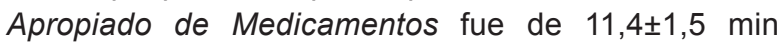
por fármaco. Para las tres evaluaciones restantes (subprescripción, reacciones adversas a medicamentos y adherencia) el tiempo promedio fue de $7,3 \pm 1,7$

Tabla 5. Evaluación de la adherencia a la farmacoterapia. Instrumento utilizado: cuestionario de Morisky y Green ${ }^{(12,13)}$.

\begin{tabular}{ccc}
\hline Puntaje & $\mathbf{n}$ & $\%$ \\
\hline $\mathbf{0}$ & 37 & $37,0 \%$ \\
$\mathbf{1}$ & 15 & $15,0 \%$ \\
$\mathbf{2}$ & 25 & $25,0 \%$ \\
$\mathbf{3}$ & 23 & $23,0 \%$ \\
Total & 100 & $100 \%$ \\
\hline
\end{tabular}

Media: 1,34 DS=1.20 (RANGO: 0 A 3)

Score de adherencia $=$ suma total de puntos por las cuatro preguntas. Interpretación: Score 0 = alta, Score 1-2 = Media, Score 3-4 = Baja min. La media de la estancia hospitalaria fue de 11,98 días (rango: 4 a 54) y la mortalidad fue de $10 \%$ durante la hospitalización.

\section{DISCUSIÓN}

En la presente investigación se encontró que los fármacos con mayores RPM de sobreprescripción y disprescripción fueron: nimodipino para demencia vascular ${ }^{(18)}$, indicación que no figura en las guías de práctica clínica, ranitidina sin especificación de duración de la terapia, uso de aspirina a dosis analgésicas y antiinflamatorias (existiendo otros antiinflamatorios no esteroides con mejor perfil de seguridad en adultos mayores), digoxina a dosis no corregidas para adultos mayores y warfarina por no regulación posológica oportuna para lograr un nivel adecuado de INR. En relación a los PRM por la subutilización de medicamentos, se encontró con mayor frecuencia la no prescripción de warfarina o aspirina en pacientes con fibrilación auricular $(21 \%)$, no utilización de beta bloqueadores en pacientes con antecedentes de infarto agudo de miocardio (59\%) y no administración de inhibidores de la enzima angiotensina convertasa en pacientes con falla cardiaca (10\%). La no adherencia a la farmacoterapia alcanzó el $63 \%$ y se correlacionó con el mayor número de fármacos $(p<0,001)$. El $24 \%$ de los pacientes tuvieron reacciones 
Tabla 6. Reacciones adversas medicamentosas en adultos mayores al momento de ser hospitalizados. Instrumento utilizado: algoritmo de Karch y Lasagna ${ }^{(16)}$.

\begin{tabular}{|c|c|}
\hline Reacción adversa medicamentosa & $\begin{array}{l}\text { Pacientes } \\
n\end{array}$ \\
\hline Hemorragia Digestiva alta por antiinflamatorios no esteroideos: diclofenanco (2), aspirina >500mg/dia (3), ibuprofeno (1) & 6 \\
\hline $\begin{array}{l}\text { Sangrado asociado a sobreanticoagulacion con warfarina: Sangrado mayor * con hemorragia digestiva alta (1), san- } \\
\text { grado menor (6), fatales }(0)\end{array}$ & 7 \\
\hline Hipoglicemia inducida por fármacos: glibenclamida (2), insulina NPH (1) & 3 \\
\hline Intoxicación digitálica & 3 \\
\hline $\begin{array}{l}\text { Trastornos de ritmo cardiaco con descompensación hemodinámica inducido por fármacos: Fenitoina (1), propranolol } \\
\text { (1), salbutamol (1), metildopa (1) }\end{array}$ & 4 \\
\hline Estreñimiento inducido por fármaco (nifedipino) & 1 \\
\hline Total & 24 \\
\hline
\end{tabular}

adversas medicamentosas que motivaron la hospitalización.

Hanlon y col. investigaron los PRM en adultos mayores frágiles hospitalizados, según el Uso Apropiado de Medicamentos (Medication Appropriatenes slndex o MAI) (19), encontrando que el $91,9 \%$ de los pacientes tenían al menos una medicación con más de un criterio inadecuado. Los problemas más frecuentemente fueron el uso de medicación muy costosa (70\%), instrucciones poco prácticas para la toma de los medicamentos $(55,2 \%)$ y posología incorrecta (50,9\%); por otro lado, el $8,9 \%$ de los fármacos de los regímenes terapéuticos del $42 \%$ de los pacientes no tenían una indicación precisa. En otro aspecto, Higashi y col. utilizaron indicadores del Assessing Care of Vulnerable Elders (ACOVE) ${ }^{(14)}$ para evaluar la calidad de prescripción farmacológica en adultos vulnerables ambulatorios; los principales problemas de subutilización de fármacos fueron: betabloqueadores para pacientes con antecedentes de infarto de miocardio (47\%), aspirina para pacientes diabéticos (59\%), inhibidores ECA para pacientes con insuficiencia cardiaca (25\%) y profilaxis de warfarina o aspirina en pacientes con fibrilación auricular $(6 \%)^{(14)}$.

Según la revisión sistemática de Hughes ${ }^{(20)}$, la prevalencia de no adherencia a la terapia farmacológica en los adultos mayores varía de 38 al $57 \%$ (promedio 45\%) con $32 \%$ en adultos mayores hospitalizados.

En relación con reacciones adversas medicamentosas causantes de hospitalización en adultos mayores, Franceschi y col. en el año 2008 encontraron una prevalencia de $5,8 \%$ en una unidad geriátrica de un hospital italiano; los problemas más frecuentes fueron de orden gastrointestinal, hemorrágico y cardiovascular y entre los fármacos implicados estuvieron antiinflamatorios no esteroideos, digoxina y warfarina (21). En el Perú, en el año 2005, se evaluó la calidad de la prescripción farmacológica en pacientes adultos mayores frágiles, por medio del Medication Appropriateness Index (MAI), y se encontró que el $90,8 \%$ de los pacientes tuvo al menos un problema inadecuado en alguno de los diez criterios del instrumento utilizado ${ }^{(22)}$; sin embargo, no se evaluó la adherencia ni el fenómeno de subutilización de fármacos. Ortiz y col. ${ }^{(23)}$ evidenciaron que el $5,5 \%$ de las hospitalizaciones en los servicios de medicina interna del Hospital Nacional Arzobispo Loayza de Lima, se asociaron con eventos adversos relacionados a medicamentos en adultos mayores, encontrándose la hipoglicemia inducida por medicamentos como la causa más frecuente $(70 \%)$. Otro estudio, realizado en el Hospital Nacional Cayetano Heredia en Lima ${ }^{24}$, halló un $19,2 \%$ de prescripciones potencialmente inadecuadas en adultos mayores. Se utilizó como instrumento de evaluación los criterios explícitos de Beers, una lista de medicamentos con una relación beneficio/ riesgo significativamente bajo.

El diseño del presente estudio fue enfocado para mostrar la factibilidad de una metodología para el diagnóstico de PRM en pacientes adultos mayores al momento de ser hospitalizados, por consiguiente, presenta limitaciones en los hallazgos de prevalencia de los PRM. La prevalencia de subutilización de medicamentos puede ser sesgada por la selección de pacientes con medicación habitual mayor de un medicamento. Por otro lado, los hallazgos sobre frecuencia de PRM, no pueden ser extrapolados a pacientes adultos mayores ambulatorios ni hospitalizados en otros centros hospitalarios de menor nivel de complejidad en el Perú. El hospital donde se realizó la investigación es un centro de referencia nacional donde generalmente se 
hospitalizan pacientes transferidos de otros centros hospitalarios de menor complejidad, por tratarse de pacientes graves o complicados. Es necesario investigar la prevalencia e incidencia de PRM en pacientes adultos mayores hospitalizados y ambulatorios. El presente estudio, tendría el valor de proporcionar una metodología con instrumentos validados para este objetivo.

En conclusión, la presente investigación encuentra que el diagnóstico de los PRM en adultos mayores al momento de ser hospitalizados, utilizando instrumentos validados, es de gran utilidad y debería formar parte de la valoración geriátrica integral en el adulto mayor, especialmente de pacientes geriátricos polimedicados.

\section{AGRADECIMIENTOS}

A los médicos asistentes y residentes del Servicio de Geriatría del Hospital Almenara.

\section{Fuentes de Financiamiento}

Instituto de Investigación de la Universidad San Martin de Porres. Lima - Perú. Código E10012008019

\section{Conflictos de Interés}

Los autores declaran no tener conflictos de interés en la publicación del presente artículo.

\section{REFERENCIAS BIBLIOGRÁFICAS}

1. Brodie DC.Drug Use Control: keystone to pharmaceutical service. Drug IntellClin Pharm. 1986;2:116-7.

2. Comité del Segundo Consenso de Granada sobre Problemas Relacionados con Medicamentos. Segundo Consenso de Granada sobre problemas relacionados con medicamentos. ArsPharmaceutica. 2002;43:179-187.

3. Spinewine A, Schmader KE, Barber N, Hughes C, Lapane KL, Swine $\mathbf{C}$ et al.Appropriate prescribing in elderly people: how well can it be measured and optimised? Lancet 2007;370:173-84.

4. Spinewine A. Appropriate use of medicines in care of theelderly-Factors underlying inappropriateness, and impact of the clinical pharmacist [tesis doctoral]. Louvain-la-Neuve, Bélgica: Université CatholiqueLouvain Université; 2006.

5. Rochon PA, Gurwitz JH. Prescribing for seniors: neither too much nor too little. JAMA. 1999;282:113-5.

6. Katz S, Ford AB, Moskowitz RW, Jackson BA, Jaffe MW.Studies of illness in the aged. The index of ADL: A standardized measure of biological and psychosocial function. JAMA 1963;185:914-9.

7. Folstein MF, Folstein SE, McHugh PR. "Mini-mental State", a practical method for grading the cognitive state of patients for the clinician. JPsychiatrRes. 1975;12(3):189-98.

8. Charlson ME, Pompei P, Ales KL, McKenzie CR. A new method for classifying prognotic in longitudinal studies: development and validation. J Chronic Dis. 1987;40(5):373-83.
9. Hanlon JT, Schmader KE, Samsa GP, Weinberger M, Uttech KM, Lewis IKet al. A method for assessing drug therapy appropriateness. J ClinEpidemiol. 1992;45(10):1045-51.

10. EsSalud. Petitorio Farmacológico2006. Lima: EsSalud; 2006. pp.1-120. Disponible en: http://www.essalud.gob.pe/ petito/petitorio2006.pdf

11. Veterans Affairs Medication Classification System. USP drug information. $20^{\text {th }}$ ed. Appendix IV. Rockville, MD: Micromedex Thompson Healthcare; 2000. p. 3220-37

12. Morisky DE, Green LW, Levine DM. Concurrent and predictive validity of a selfreported measure of medication adherence. Med Care. 1986;24:67-74.

13. Val Jiménez A, Amorós G, Martínez $P$, Ferández $M L$, León M. Estudio descriptivo delcumplimiento del tratamiento farmacológico antihipertensivo y validación del testMorisky y Green. Aten Primaria. 1992;10:767-70.

14. Higashi T, Shekelle PG, Solomon DH, Knight EL, Roth C, Chang JT et al. The quality of pharmacologic care for vulnerable older patients. Ann Intern Med. 2004;140(9):714-20.

15. Lipton HL, Bero LA, Bird JA. Under medication among geriatric outpatients: results of a randomized controlled trial. Ann Rev Gerontol Ger. 1992;12:95-108.

16. KarchFE, LasagnaL. Toward the operational definition of adverse drug reactions. Clin Pharmac Ther. 1977;21:24754.

17. Graves T,Hanlon JT, Schmader KE, Landsman PB, Samsa GP, Pieper CF et al. Adverse events after discontinuing medications in elderly outpatients. Arch Intern Med. 1997; 157:2205-10

18. López-Arrieta B.Nimodipinapara la demencia degenerativa primaria, mixta y vascular. La Biblioteca Cochrane Plus. 2008;(2):CD000147. Disponible en: http://www.update-software.com

19. Hanlon JT, Artz MB, Pieper CF, Lindblad Cl, Sloane RJ, Ruby $\mathbf{C M}$ et al. Inappropriate medication use among frail elderly inpatients. Ann Pharmacother. 2004;1:9-14.

20. Hughes $\mathbf{C M}$. Medication non-adherence in the elderly: how big is the problem? Drugs Aging. 2004;21(12):793-811.

21. Franceschi M, Scarcelli C, Niro V, Seripa D, Pazienza AM, Pepe $\mathbf{G}$ et al. Prevalence, clinical features and avoidability of adverse drug reactions as cause of admission to a geriatric unit: a prospective study of 1756 patients. Drug Saf. 2008;31(6):545-56.

22. Oscanoa T, Lira G. Calidad de prescripción de medicamentos en pacientes geriátricos. An Fac Med. 2005;66(2):195202.

23. Ortiz P, Juárez M, Valdivia M, Varela L.Hospitalización por eventos adversos relacionados a medicamentos en adultos mayores. Rev Soc Peru Med Interna. 2009;22(2):53-6.

24. Córdova V., Chavez H., Varela L. Características de la prescripción farmacológica en pacientes adultos mayores hospitalizados. Diagnostico. 2005;44(4):151-9.

Correspondencia: Dr. Teodoro Julio Oscanoa

Dirección: Hospital Almenara. Av. Grau 800. Lima 13, Perú.

Teléfono: (511) 324-2983 Anexo 4085

Correo electrónico: teodoro.oscanoa@essalud.gob.pe 